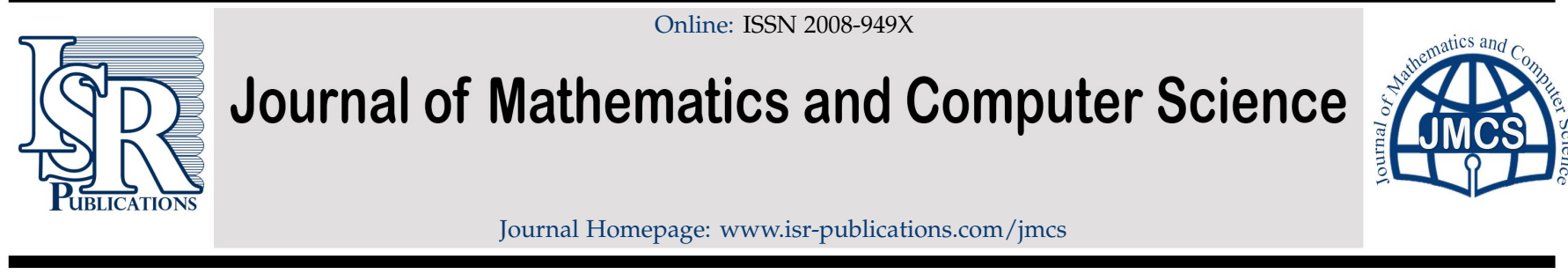

\title{
New group iterative schemes for solving the two- dimensional anomalous fractional sub-diffusion equation
}

\author{
Ajmal Ali ${ }^{\mathrm{a}, *}$, Muhammad Abbas ${ }^{\mathrm{b}}$, Tayyaba Akram ${ }^{\mathrm{c}}$ \\ ${ }^{a}$ Department of Mathematics, Virtual University of Pakistan, Lahore, Pakistan. \\ ${ }^{b}$ Departemnt of Mathematics, University of Sargodha, Sargodha, Pakistan. \\ 'School of Mathematical Sciences, Universiti Sains Malaysia, Penang, Malaysia.
}

\begin{abstract}
In this paper, new group iterative schemes are developed for the numerical solution of two-dimensional anomalous fractional sub-diffusion equation subject to specific initial and Dirichlet boundary conditions. The new group relaxation iterative schemes are derived from the combination of standard and rotated (skewed) five-point modified implicit finite difference approximations. The results derived from the conducted numerical experiments show that fractional explicit de-coupled group (FEDG) iterative method has a significantly less computational cost in terms of CPU-timings as compared to the other iterative schemes, without threatening compromising accuracies.
\end{abstract}

Keywords: Riemann-Liouville fractional derivative, fractional implicit standard point, fractional implicit rotated point, anomalous fractional sub-diffusion equation.

2020 MSC: 65N06, 65M06, 65M12.

(C)2021 All rights reserved.

\section{Introduction}

In recent years, there has been a growing interest in the field of fractional differential equations (FDEs). FDEs have fascinated growing significance because they have several applications in different fields of science and engineering [19,27]. Many types of FDEs cannot be solved analytically, therefore a number of mathematical methods, techniques, and approximations have been proposed for solving partial differential equation of fractional order due to their importance in representing the various physical problems $[1,2,7,8,20,21]$. However, the study of exact analytic solution of some types FDEs are also present in literature $[9,18,24,25]$. Fractional anomalous diffusion equations are a special type of fractional subdiffusion equation and are a class of important FDEs. Several mathematicians have solved such type of research problems and their modified forms by different techniques. Li and Wang [22] suggested an improved efficient difference method for modified anomalous sub-diffusion equation with a nonlinear source term. They utilized shifted Grunwald-Letnikov fractional derivative instead of Riemann-Liouville derivative and second-order interpolation formula for nonlinear source term. Zhuang et al. [28] proposed

\footnotetext{
*Corresponding author

Email address: ajmal@vu.edu.pk (Ajmal Ali)
}

doi: $10.22436 /$ jmcs.022.02.03

Received: 2020-03-31 Revised: 2020-04-23 Accepted: 2020-05-24 
new solution and analytic techniques for anomalous sub-diffusion equation by using implicit difference scheme and they proved the stability and convergence by energy method. Sokolov and Klafter [26] proposed a model that differentiate procedure to become less anomalous as time growth by the addition of a secondary time fractional derivative acting on a diffusion operator. In this paper, we assume such type of model and will applying our grouping strategies. Consider the following two-dimensional time-fractional anomalous fractional sub-diffusion equation together withe source term,

$$
\frac{\partial u(x, y, t)}{\partial t}=\frac{\partial^{1-\alpha}}{\partial t^{1-\alpha}}\left[\frac{\partial^{2} u(x, y, t)}{\partial x^{2}}+\frac{\partial^{2} u(x, y, t)}{\partial y^{2}}\right]+f(x, y, t)
$$

where, $0<\alpha<1$ and $\frac{\partial^{1-\alpha}}{\partial t^{1-\alpha}}$ is the the Riemann-Liouville fractional derivatives of fractional order $1-\alpha$ with the following initial and boundary conditions,

$$
u(x, y, 0)=g(x, y), \quad u(0, y, t)=g_{1}(y, t), u(L, y, t)=g_{2}(y, t), \quad u(x, 0, t)=g_{3}(x, t), u(x, L, t)=g_{4}(x, t),
$$

in the region $\Omega=\{(x, y, t) / 0<x, y<L, 0 \leqslant t \leqslant T\}$.

The Riemann-Liouville fractional derivatives are defined as follows,

$$
\frac{\partial^{1-\alpha}}{\partial t^{1-\alpha}} \mathfrak{u}(x, y, t)=\frac{1}{\Gamma(\alpha)} \frac{\partial}{\partial t} \int_{0}^{t} \frac{u(x, y, \xi)}{(t-\xi)^{1-\alpha}} d \xi=\frac{\partial}{\partial t} I_{0}^{\alpha} u(x, y, t)
$$

Here

$$
I_{0}^{\alpha} u(x, y, t)=\frac{1}{\Gamma(\alpha)} \int_{0}^{t} \frac{u(x, y, \xi)}{(t-\xi)^{1-\alpha}} d \xi
$$

is the Riemann-Liouville integral of fractional order $\alpha$. In this paper, we use the following two Lemmas.

Lemma 1.1. If $\mathrm{u}(\mathrm{t}) \in \mathrm{C}^{1}[0, \mathrm{~T}]$, then the Riemann-Liouville fractional integral of order $0<\gamma<1$ is defined as follows,

$$
\mathrm{I}_{0}^{\gamma} \mathrm{u}\left(\mathrm{t}_{\mathrm{k}}\right)=\frac{\tau^{\gamma}}{\Gamma(\gamma+1)} \sum_{s=0}^{k-1} \mathrm{~b}_{s}^{\gamma} \mathrm{u}\left(\mathrm{t}_{\mathrm{k}-\mathrm{s}}\right)+\mathrm{R}_{\mathrm{k}}^{\gamma},
$$

where, $\mathrm{b}_{\mathrm{s}}^{\gamma}=(\mathrm{s}+1)^{\gamma}-\mathrm{s}^{\gamma}, \mathrm{s}=0,1,2 \cdots \mathrm{k}, \quad\left|\mathrm{R}_{\mathrm{k}}^{\gamma}\right| \leqslant \mathrm{C} \tau \mathrm{b}_{\mathrm{k}}^{\gamma}$.

Lemma 1.2. The coefficients $b_{\mathrm{k}}^{\gamma}$ in Lemma 1, satisfy the following properties

(a) $\mathrm{b}_{0}^{\gamma}=1, \mathrm{~b}_{\mathrm{k}}^{\gamma}>0, \quad \forall \mathrm{k}=0,1,2, \ldots$;

(b) $\mathrm{b}_{\mathrm{k}-1}^{\gamma}>\mathrm{b}_{\mathrm{k}}^{\gamma}, \forall \mathrm{k}=0,1,2, \ldots$;

(c) For any positive constant $\mathrm{C}>0$ such that $\tau \leqslant \mathrm{Cb}_{\mathrm{k}}^{\gamma} \tau^{\gamma}, \forall \mathrm{k}=0,1,2, \ldots$;

(d) $\sum_{s=0}^{k} b_{s}^{\gamma} \tau^{\gamma}=(k+1)^{\gamma} \leqslant T^{\gamma}, \quad \forall 0<\gamma<1$.

The discretization of our proposed research problem in terms of partial differential equation by utilizing the finite difference approximations, generates a sparse system of simultaneous linear equations. For the solution of this linear system, iterative methods are very suitable. In the literature, many iterative methods have been suggested by various authors for solving the linear system. Moreover, several studies on iterative methods have been proposed to speed up the convergence rate in solving sparse linear system. One of them is the development of group iterative methods that possess the capabilities to reduce the convergence timings of the iterative process.

Since 1980s, the group iterative schemes in combination with the finite difference have been utilized for the numerical solution of several types of two-dimensional PDEs in literature. Evans (1985) [14] utilized Explicit Group (EG) iterative method by designing 4-point block iterative scheme for 2D Poisson problem. Based on this ideology, a series of EG iterative schemes have extensively been investigated by Evans and Yousif (1986) [17], Evans and Sahimi (1988) [16], Evans and Hasan (2003) [15] and Kew and Ali (2015) [23] for the numerical solution of various types of PDEs. Since these grouping strategies on integer derivative 
for PDEs work well, therefore the efforts are now being made to apply these techniques on FDEs. In this case, the preliminary work has been done by Balasim and Ali (2016-17) [10-12] on two-dimensional timefractional diffusion and advection-diffusion PDEs, by utilizing Caputo's fractional derivative, where the time-fractional derivative of order $\alpha$ lies between 0 and 1 . In addition, the most recent work by Ali and Ali (2018-19) [3-5] on two-dimensional time-fractional wave, damped wave and hyperbolic telegraph PDEs, by using the same definition of Caputo's fractional derivative, where the order of time-fractional derivative $\alpha \in(1,2)$. In this paper, we step forward towards the numerical solution of more complicated 2D timefractional anomalous sub-diffusion equation by applying by Riemann-Liouville fractional derivative and grouping methods, and results were found according to our expectation.

The origination of the paper will be as follows. We will discretize our proposed problem by applying the implicit finite difference approximation and derive FISP iterative scheme in Section 2. In Section 3, FEG iterative scheme will be derived by applying a group of 4-points in the solution domain. FIRP iterative scheme will be constructed by rotating the $x-y$ axis clockwise through an angle of $45^{\circ}$ to the standard mesh in Section 4. In Section 5, FEDG iterative scheme will be established based on FIRP iterative scheme. The numerical experiments and conclusion will be present in Sections 6 and 7, respectively.

\section{Fractional implicit standard point (FISP) iterative scheme}

Discretize the solution domain by defining the same step sizes $h$ in both $x$ and $y$ directions, i.e., $\Delta x=\Delta y=h$ as $h=\frac{L}{n}$ and $\tau=\frac{T}{l}$, for the positive integers $\mathrm{n}$ and $l$, for the space and time respectively. The grid points for the space interval $[0,1]$ are designated as $x_{i}=i$ h and $y_{i}=j h, i, j=0,1,2, \ldots, n$, and grid points for time are chosen as $t_{k}=k \tau, k=0,1,2, \ldots, l$.

We utilize Riemann-Liouville derivative for time-fractional case and implicit difference approximation for second-order space derivatives on the right side, while forward difference approximation with respect to time on left side of Eq. (1.1). Riemann-Liouville integral operator defined in Lemma (1.1) will automatically be utilized when we use Riemann-Liouville fractional derivative for solving the 2D anomalous fractional sub-diffusion equation.

In this way, the following fractional implicit standard point (FISP) iterative scheme at the point $\left(x_{i}, y_{j}, t_{k+1}\right)$ is obtained,

$$
u_{i, j}^{k+1}-u_{i, j}^{k}=r_{1} \sum_{s=0}^{k-1} b_{s}^{(\alpha)} \delta_{x}^{2}\left(u_{i, j}^{k-s+1}-u_{i, j}^{k-s}\right)+r_{2} \sum_{s=0}^{k-1} b_{s}^{(\alpha)} \delta_{y}^{2}\left(u_{i, j}^{k-s+1}-u_{i, j}^{k-s}\right)+\tau f_{i, j}^{k+1}+R_{i, j, k}^{\alpha}
$$

for $i, j=1,2,3, \ldots, n-1, k=0,1,2,3, \ldots, l$.

Here, the operators $\delta_{x}^{2}$ and $\delta_{y}^{2}$ are defined as,

$$
\delta_{x}^{2} u_{i, j}^{k+1}=u_{i-1, j}^{k+1}-2 u_{i, j}^{k+1}+u_{i+1, j}^{k+1} \quad \text { and } \quad \delta_{y}^{2} u_{i, j}^{k+1}=u_{i, j-1}^{k+1}-2 u_{i, j}^{k+1}+u_{i, j+1}^{k+1} .
$$

Moreover, $r_{1}=\frac{\tau^{\alpha} k_{1}}{(\Delta x)^{2} \Gamma(1+\alpha)}, r_{2}=\frac{\tau^{\alpha} k_{2}}{(\Delta y)^{2} \Gamma(1+\alpha)}$,

$$
\left|R_{i, j, k}^{\alpha}\right| \leqslant C b_{k}^{(\alpha)} \tau^{\alpha}\left(\tau+\tau(\Delta x)^{2}\right) .
$$

An alternative form of the above equation is

$$
\begin{aligned}
u_{i, j}^{k+1}= & 1 /\left(1-r_{1} \delta_{x}^{2}-r_{2} \delta_{y}^{2}\right)\left[\left\{1+r_{1}\left(b_{1}^{(\alpha)}-1\right) \delta_{x}^{2}+r_{2}\left(b_{1}^{(\alpha)}-1\right) \delta_{y}^{2}\right\} u_{i, j}^{k}\right. \\
& \left.-\left\{b_{k}^{(\alpha)}\left(r_{1} \delta_{x}^{2}+r_{2} \delta_{y}^{2}\right)\right\} u_{i, j}^{1}+\sum_{s=1}^{k-1}\left(b_{s-1}^{(\alpha)}-b_{s}^{(\alpha)}\right)\left(r_{1} \delta_{x}^{2}+r_{2} \delta_{y}^{2}\right) u_{i, j}^{k-s}+\tau f_{i, j}^{k+1}\right] .
\end{aligned}
$$


Applying the operators $\delta_{x}^{2}$ and $\delta_{y}^{2}$ on $u_{i, j}^{k+1}$ and $u_{i, j}^{k}$, we get the following expression,

$$
\begin{aligned}
\left(1+2 r_{1}+2 r_{2}\right) u_{i, j}^{k+1}= & \left(r_{1}\right)\left(u_{i-1, j}^{k+1}+u_{i+1, j}^{k+1}\right)+\left(r_{2}\right)\left(u_{i, j-1}^{k+1}+u_{i, j+1}^{k+1}\right) \\
& +\left\{r_{1}\left(b_{1}^{(\alpha)}-1\right)\right\}\left(u_{i-1, j}^{k}+u_{i+1, j}^{k}\right)+\left\{r_{2}\left(b_{1}^{(\alpha)}-1\right)\right\}\left(u_{i, j-1}^{k}+u_{i, j+1}^{k}\right) \\
& +\left\{1-2 r_{1}\left(b_{1}^{(\alpha)}-1\right)-2 r_{2}\left(b_{1}^{(\alpha)}-1\right)\right\} u_{i, j}^{k}-\left\{b_{k}^{(\alpha)}\left(r_{1} \delta_{x}^{2}+r_{2} \delta_{y}^{2}\right)\right\} u_{i, j}^{1} \\
& \left.+\sum_{s=1}^{k-1}\left(b_{s-1}^{(\alpha)}-b_{s}^{(\alpha)}\right)\left(r_{1} \delta_{x}^{2}+r_{2} \delta_{y}^{2}\right) u_{i, j}^{k-s}+\tau f_{i, j}^{k+1}\right],
\end{aligned}
$$

for all $i, j=1,2,3, \ldots, n-1, k=0,1,2,3, \ldots, l$ with initial and boundary conditions,

$$
u_{i, j}^{0}=g\left(x_{i}, y_{j}\right), \quad u_{0, j}^{k}=g_{1}\left(y_{j}, t_{k}\right), u_{L, j}^{k}=g_{2}\left(y_{j}, t_{k}\right), \quad u_{i, 0}^{k}=g_{3}\left(x_{i}, t_{k}\right), u_{i, L}^{k}=g_{4}\left(x_{i}, t_{k}\right),
$$

$0<x, y<L, 0 \leqslant t \leqslant T$.

\section{Fractional explicit group (FEG) iterative scheme}

The fractional explicit group (FEG) iterative scheme can be formulated by applying Eq. (2.1) on a group of four points of the solution domain. This will result in a $4 \times 4$ system of equations as follows:

$$
\left(\begin{array}{cccc}
k_{1} & k_{2} & 0 & k_{3} \\
k_{2} & k_{1} & k_{3} & 0 \\
0 & k_{3} & k_{1} & k_{2} \\
k_{3} & 0 & k_{2} & k_{1}
\end{array}\right)\left(\begin{array}{c}
u_{i, j}^{k+1} \\
u_{i+1, j}^{k+1} \\
u_{i+1, j+1}^{k+1} \\
u_{i, j+1}^{k+1}
\end{array}\right)=\left(\begin{array}{c}
\left(r_{1}\right) u_{i-1, j}^{k+1}+\left(r_{2}\right) u_{i, j-1}^{k+1}+T_{1} \\
\left(r_{1}\right) u_{i+2, j}^{k+1}+\left(r_{2}\right) u_{i+1, j-1}^{k+1}+T_{2} \\
\left(r_{1}\right) u_{i+2, j+1}^{k+1}+\left(r_{2}\right) u_{i+1, j+2}^{k+1}+T_{3} \\
\left(r_{1}\right) u_{i-1, j+1}^{k+1}+\left(r_{2}\right) u_{i, j+2}^{k+1}+T_{4}
\end{array}\right)
$$

where, $k_{1}=1+2 r_{1}+2 r_{2}, k_{2}=-r_{1}, k_{3}=-r_{2}$,

$$
\begin{aligned}
& \mathrm{T}_{1}=\left\{r_{1}\left(b_{1}^{(\alpha)}-1\right)\right\}\left(u_{i-1, j}^{k}+u_{i+1, j}^{k}\right)+\left\{r_{2}\left(b_{1}^{(\alpha)}-1\right)\right\}\left(u_{i, j-1}^{k}+u_{i, j+1}^{k}\right) \\
&+\left\{1-2 r_{1}\left(b_{1}^{(\alpha)}-1\right)-2 r_{2}\left(b_{1}^{(\alpha)}-1\right)\right\} u_{i, j}^{k}-\left\{b_{k}^{(\alpha)}\left(r_{1} \delta_{x}^{2}+r_{2} \delta_{y}^{2}\right)\right\} u_{i, j}^{1} \\
&+\sum_{s=1}^{k-1}\left(b_{s-1}^{(\alpha)}-b_{s}^{(\alpha)}\right)\left(r_{1} \delta_{x}^{2}+r_{2} \delta_{y}^{2}\right) u_{i, j}^{k-s}+\tau f_{i, j}^{k+1}, \\
& T_{2}=\left\{r_{1}\left(b_{1}^{(\alpha)}-1\right)\right\}\left(u_{i, j}^{k}+u_{i+2, j}^{k}\right)+\left\{r_{2}\left(b_{1}^{(\alpha)}-1\right)\right\}\left(u_{i+1, j-1}^{k}+u_{i+1, j+1}^{k}\right) \\
&+\left\{1-2 r_{1}\left(b_{1}^{(\alpha)}-1\right)-2 r_{2}\left(b_{1}^{(\alpha)}-1\right)\right\} u_{i+1, j}^{k}-\left\{b_{k}^{(\alpha)}\left(r_{1} \delta_{x}^{2}+r_{2} \delta_{y}^{2}\right)\right\} u_{i+1, j}^{1} \\
& \\
&+\sum_{s=1}^{k-1}\left(b_{s-1}^{(\alpha)}-b_{s}^{(\alpha)}\right)\left(r_{1} \delta_{x}^{2}+r_{2} \delta_{y}^{2}\right) u_{i+1, j}^{k-s}+\tau f_{i+1, j}^{k+1} \\
& T_{3}=\left\{r_{1}\left(b_{1}^{(\alpha)}-1\right)\right\}\left(u_{i, j+1}^{k}+u_{i+2, j+1}^{k}\right)+\left\{r_{2}\left(b_{1}^{(\alpha)}-1\right)\right\}\left(u_{i+1, j}^{k}+u_{i+1, j+2}^{k}\right) \\
&+\left\{1-2 r_{1}\left(b_{1}^{(\alpha)}-1\right)-2 r_{2}\left(b_{1}^{(\alpha)}-1\right)\right\} u_{i+1, j+1}^{k}-\left\{b_{k}^{(\alpha)}\left(r_{1} \delta_{x}^{2}+r_{2} \delta_{y}^{2}\right)\right\} u_{i+1, j+1}^{1} \\
& \\
&+\sum_{s=1}^{k-1}\left(b_{s-1}^{(\alpha)}-b_{s}^{(\alpha)}\right)\left(r_{1} \delta_{x}^{2}+r_{2} \delta_{y}^{2}\right) u_{i+1, j+1}^{k-s}+\tau f_{i+1, j+1}^{k+1} \\
& T_{4}=\left\{r_{1}\left(b_{1}^{(\alpha)}-1\right)\right\}\left(u_{i-1, j+1}^{k}+u_{i+1, j+1}^{k}\right)+\left\{r_{2}\left(b_{1}^{(\alpha)}-1\right)\right\}\left(u_{i, j}^{k}+u_{i, j+2}^{k}\right) \\
&+\left\{1-2 r_{1}\left(b_{1}^{(\alpha)}-1\right)-2 r_{2}\left(b_{1}^{(\alpha)}-1\right)\right\} u_{i, j+1}^{k}-\left\{b_{k}^{(\alpha)}\left(r_{1} \delta_{x}^{2}+r_{2} \delta_{y}^{2}\right)\right\} u_{i, j+1}^{1} \\
&+\sum_{s-1}^{k}\left(b_{s-1}^{(\alpha)}-b_{s}^{(\alpha)}\right)\left(r_{1} \delta_{x}^{2}+r_{2} \delta_{y}^{2}\right) u_{i, j+1}^{k-s}+\tau f_{i, j+1}^{k+1} .
\end{aligned}
$$


Rewrite matrix Eq. (3.1) as,

$$
\left(\begin{array}{c}
u_{i, j}^{k+1} \\
u_{i+1, j}^{k+1} \\
u_{i+1, j+1}^{k+1} \\
u_{i, j+1}^{k+1}
\end{array}\right)=\frac{1}{M}\left(\begin{array}{llll}
p_{1} & p_{2} & p_{3} & p_{4} \\
p_{2} & p_{1} & p_{4} & p_{3} \\
p_{3} & p_{4} & p_{1} & p_{2} \\
p_{4} & p_{3} & p_{2} & p_{1}
\end{array}\right)\left(\begin{array}{c}
\left(r_{1}\right) u_{i-1, j}^{k+1}+\left(r_{2}\right) u_{i, j-1}^{k+1}+T_{1} \\
\left(r_{1}\right) u_{i+2, j}^{k+1}+\left(r_{2}\right) u_{i+1, j-1}^{k+1}+T_{2} \\
\left(r_{1}\right) u_{i+2, j+1}^{k+1}+\left(r_{2}\right) u_{i+1, j+2}^{k+1}+T_{3} \\
\left(r_{1}\right) u_{i-1, j+1}^{k+1}+\left(r_{2}\right) u_{i, j+2}^{k+1}+T_{4}
\end{array}\right)
$$

where,

$$
\begin{aligned}
& M=\left(1+2 r_{1}+2 r_{2}\right)^{4}-2\left(1+2 r_{1}+2 r_{2}\right)^{2}\left[\left(r_{1}\right)^{2}+\left(r_{2}\right)^{2}\right]+\left[\left(r_{1}\right)^{2}-\left(r_{2}\right)^{2}\right]^{2}, \\
& p_{1}=\left(1+2 r_{1}+2 r_{2}\right)\left[\left(1+2 r_{1}+2 r_{2}\right)^{2}-\left(r_{1}\right)^{2}-\left(r_{2}\right)^{2}\right], \\
& p_{2}=\left(r_{1}\right)\left[\left(1+2 r_{1}+2 r_{2}\right)^{2}-\left(r_{1}\right)^{2}+\left(r_{2}\right)^{2}\right], \\
& p_{3}=2\left(1+2 r_{1}+2 r_{2}\right)\left(r_{1}\right)\left(r_{2}\right), \\
& p_{4}=\left(r_{2}\right)^{2}\left[\left(1+2 r_{1}+2 r_{2}\right)^{2}+\left(r_{1}\right)^{2}-\left(r_{2}\right)^{2}\right] .
\end{aligned}
$$

The FEG iterative method decreases the computational cost by reducing the total number of arithmetic operations used in the iterative procedure. It earlier stored all the arithmetic operations of each 4-point calculations in the form of a group and afterward this group treated as a single element in the entire iterative process generated by Eq. (3.2) by reducing CPU-timings.

\section{Fractional implicit rotated point (FIRP) iterative scheme}

Another implicit difference scheme can be constructed by rotating Eq. (2.1) an angle $45^{\circ}$ to the standard mesh [6]. We have the following fractional implicit rotated point (FIRP) iterative scheme,

$$
\begin{aligned}
\left(1+r_{1}+r_{2}\right) u_{i, j}^{k+1}= & \frac{1}{2}\left(r_{1}\right)\left(u_{i-1, j+1}^{k+1}+u_{i+1, j-1}^{k+1}\right)+\frac{1}{2}\left(r_{2}\right)\left(u_{i+1, j+1}^{k+1}+u_{i-1, j-1}^{k+1}\right) \\
& +\frac{1}{2}\left\{r_{1}\left(b_{1}^{(\alpha)}-1\right)\right\}\left(u_{i-1, j+1}^{k}+u_{i+1, j-1}^{k}\right)+\frac{1}{2}\left\{r_{2}\left(b_{1}^{(\alpha)}-1\right)\right\}\left(u_{i+1, j+1}^{k}+u_{i-1, j-1}^{k}\right) \\
& +\left\{1-r_{1}\left(b_{1}^{(\alpha)}-1\right)-r_{2}\left(b_{1}^{(\alpha)}-1\right)\right\} u_{i, j}^{k}-\left\{b_{k}^{(\alpha)}\left(r_{1} \delta_{x}^{2}+r_{2} \delta_{y}^{2}\right)\right\} u_{i, j}^{1} \\
& \left.+\sum_{s=1}^{k-1}\left(b_{s-1}^{(\alpha)}-b_{s}^{(\alpha)}\right)\left(r_{1} \delta_{x}^{2}+r_{2} \delta_{y}^{2}\right) u_{i, j}^{k-s}+\tau f_{i, j}^{k+1}\right],
\end{aligned}
$$

for all $i, j=1,2,3, \ldots, n-1, k=0,1,2,3, \ldots, l$ with initial and boundary conditions,

$$
u_{i, j}^{0}=g\left(x_{i}, y_{j}\right), \quad u_{0, j}^{k}=g_{1}\left(y_{j}, t_{k}\right), u_{L, j}^{k}=g_{2}\left(y_{j}, t_{k}\right), \quad u_{i, 0}^{k}=g_{3}\left(x_{i}, t_{k}\right), u_{i, L}^{k}=g_{4}\left(x_{i}, t_{k}\right),
$$

$0<x, y<L, 0 \leqslant t \leqslant T$.

\section{Fractional explicit de-coupled group (FEDG) iterative scheme}

Similar to FEG iterative scheme, the fractional explicit de-coupled group (FEDG) iterative scheme can be constructed by applying Eq. (4.1) on a group of four points of the solution domain. Since FEDG iterative scheme has the rotation factor due the involvement of Eq. (4.1), therefore, at the end, we got two independent set of matrix equation. In this way, we have a $4 \times 4$ system of equations of the following form:

$$
\left(\begin{array}{cccc}
l_{1} & l_{3} & 0 & 0 \\
l_{3} & l_{1} & 0 & 0 \\
0 & 0 & l_{1} & l_{2} \\
0 & 0 & l_{2} & l_{1}
\end{array}\right)\left(\begin{array}{c}
u_{i, j}^{k+1} \\
u_{i+1, j+1}^{k+1} \\
u_{i+1, j}^{k+1} \\
u_{i, j+1}^{k+1}
\end{array}\right)=\left(\begin{array}{c}
\frac{1}{2}\left(r_{1}\right)\left(u_{i-1, j+1}^{k+1}+u_{i+1, j-1}^{k+1}\right)+\frac{1}{2}\left(r_{2}\right)\left(u_{i-1, j-1}^{k+1}\right)+T_{1}^{*} \\
\frac{1}{2}\left(r_{1}\right)\left(u_{i, j+2}^{k+1}+u_{i+2, j}^{k+1}\right)+\frac{1}{2}\left(r_{2}\right)\left(u_{i+2, j+2}^{k+1}\right)+T_{2}^{*} \\
\frac{1}{2}\left(r_{2}\right)\left(u_{i, j-1}^{k+1}+u_{i+2, j+1}^{k+1}\right)+\frac{1}{2}\left(r_{1}\right)\left(u_{i+2, j-1}^{k+1}\right)+T_{3}^{*} \\
\frac{1}{2}\left(r_{2}\right)\left(u_{i-1, j}^{k+1}+u_{i+1, j+2}^{k+1}\right)+\frac{1}{2}\left(r_{1}\right)\left(u_{i-1, j+2}^{k+1}\right)+T_{4}^{*}
\end{array}\right),
$$


where, $l_{1}=1+r_{1}+r_{2}, l_{2}=-\frac{1}{2}\left(r_{1}\right), l_{3}=-\frac{1}{2}\left(r_{2}\right)$,

$$
\begin{aligned}
& \mathrm{T}_{1}^{*}=\frac{1}{2}\left\{\mathrm{r}_{1}\left(\mathrm{~b}_{1}^{(\alpha)}-1\right)\right\}\left(\mathrm{u}_{\mathrm{i}-1, j+1}^{\mathrm{k}}+\mathrm{u}_{\mathrm{i}+1, j-1}^{\mathrm{k}}\right)+\frac{1}{2}\left\{\mathrm{r}_{2}\left(\mathrm{~b}_{1}^{(\alpha)}-1\right)\right\}\left(\mathrm{u}_{\mathrm{i}+1, j+1}^{\mathrm{k}}+\mathrm{u}_{\mathrm{i}-1, j-1}^{\mathrm{k}}\right) \\
& +\left\{1-r_{1}\left(b_{1}^{(\alpha)}-1\right)-r_{2}\left(b_{1}^{(\alpha)}-1\right)\right\} u_{i, j}^{k}-\left\{b_{k}^{(\alpha)}\left(r_{1} \delta_{x}^{2}+r_{2} \delta_{y}^{2}\right)\right\} u_{i, j}^{1} \\
& +\sum_{s=1}^{k-1}\left(b_{s-1}^{(\alpha)}-b_{s}^{(\alpha)}\right)\left(r_{1} \delta_{x}^{2}+r_{2} \delta_{y}^{2}\right) u_{i, j}^{k-s}+\tau f_{i, j}^{k+1}, \\
& \mathrm{~T}_{2}^{*}=\frac{1}{2}\left\{\mathrm{r}_{1}\left(\mathrm{~b}_{1}^{(\alpha)}-1\right)\right\}\left(\mathrm{u}_{\mathrm{i}, \mathrm{j}+2}^{\mathrm{k}}+\mathrm{u}_{\mathrm{i}+2, \mathrm{j}}^{\mathrm{k}}\right)+\frac{1}{2}\left\{\mathrm{r}_{2}\left(\mathrm{~b}_{1}^{(\alpha)}-1\right)\right\}\left(\mathrm{u}_{\mathrm{i}+2, j+2}^{\mathrm{k}}+\mathrm{u}_{\mathrm{i}, \mathrm{j}}^{\mathrm{k}}\right) \\
& +\left\{1-r_{1}\left(b_{1}^{(\alpha)}-1\right)-r_{2}\left(b_{1}^{(\alpha)}-1\right)\right\} u_{i+1, j+1}^{k}-\left\{b_{k}^{(\alpha)}\left(r_{1} \delta_{x}^{2}+r_{2} \delta_{y}^{2}\right)\right\} u_{i+1, j+1}^{1} \\
& +\sum_{s=1}^{k-1}\left(b_{s-1}^{(\alpha)}-b_{s}^{(\alpha)}\right)\left(r_{1} \delta_{x}^{2}+r_{2} \delta_{y}^{2}\right) u_{i+1, j+1}^{k-s}+\tau f_{i+1, j+1}^{k+1} \\
& \mathrm{~T}_{3}^{*}=\frac{1}{2}\left\{\mathrm{r}_{1}\left(\mathrm{~b}_{1}^{(\alpha)}-1\right)\right\}\left(\mathrm{u}_{\mathrm{i}, \mathrm{j}+1}^{\mathrm{k}}+\mathrm{u}_{\mathrm{i}+2, j-1}^{\mathrm{k}}\right)+\frac{1}{2}\left\{\mathrm{r}_{2}\left(\mathrm{~b}_{1}^{(\alpha)}-1\right)\right\}\left(\mathrm{u}_{\mathrm{i}+2, j+1}^{\mathrm{k}}+\mathrm{u}_{\mathrm{i}, j-1}^{\mathrm{k}-1}\right) \\
& +\left\{1-r_{1}\left(b_{1}^{(\alpha)}-1\right)-r_{2}\left(b_{1}^{(\alpha)}-1\right)\right\} u_{i+1, j}^{k}-\left\{b_{k}^{(\alpha)}\left(r_{1} \delta_{x}^{2}+r_{2} \delta_{y}^{2}\right)\right\} u_{i+1, j}^{1} \\
& +\sum_{s=1}^{k-1}\left(b_{s-1}^{(\alpha)}-b_{s}^{(\alpha)}\right)\left(r_{1} \delta_{x}^{2}+r_{2} \delta_{y}^{2}\right) u_{i+1, j}^{k-s}+\tau f_{i+1, j}^{k+1}, \\
& \mathrm{~T}_{4}^{*}=\frac{1}{2}\left\{\mathrm{r}_{1}\left(\mathrm{~b}_{1}^{(\alpha)}-1\right)\right\}\left(\mathrm{u}_{\mathrm{i}-1, j+2}^{\mathrm{k}}+\mathrm{u}_{\mathrm{i}+1, \mathrm{j}}^{\mathrm{k}}\right)+\frac{1}{2}\left\{\mathrm{r}_{2}\left(\mathrm{~b}_{1}^{(\alpha)}-1\right)\right\}\left(\mathrm{u}_{\mathrm{i}+1, j+2}^{\mathrm{k}}+\mathrm{u}_{\mathrm{i}-1, \mathrm{j}}^{\mathrm{k}}\right) \\
& +\left\{1-r_{1}\left(b_{1}^{(\alpha)}-1\right)-r_{2}\left(b_{1}^{(\alpha)}-1\right)\right\} u_{i, j+1}^{k}-\left\{b_{k}^{(\alpha)}\left(r_{1} \delta_{x}^{2}+r_{2} \delta_{y}^{2}\right)\right\} u_{i, j+1}^{1} \\
& +\sum_{s=1}^{k-1}\left(b_{s-1}^{(\alpha)}-b_{s}^{(\alpha)}\right)\left(r_{1} \delta_{x}^{2}+r_{2} \delta_{y}^{2}\right) u_{i, j+1}^{k-s}+\tau f_{i, j+1}^{k+1} \text {. }
\end{aligned}
$$

The matrix Eq. (5.1) can be written as pair of matrix equations,

$$
\left(\begin{array}{c}
u_{i, j}^{k+1} \\
u_{i+1, j+1}^{k+1}
\end{array}\right)=\frac{1}{s_{1}}\left(\begin{array}{cc}
s_{1} & s_{2} \\
s_{2} & s_{1}
\end{array}\right)\left(\begin{array}{c}
\frac{1}{2}\left(r_{1}\right)\left(u_{i-1, j+1}^{k+1}+u_{i+1, j-1}^{k+1}\right)+\frac{1}{2}\left(r_{2}\right)\left(u_{i-1, j-1}^{k+1}\right)+T_{1}^{*} \\
\frac{1}{2}\left(r_{1}\right)\left(u_{i, j+2}^{k+1}+u_{i+2, j}^{k+1}\right)+\frac{1}{2}\left(r_{2}\right)\left(u_{i+2, j+2}^{k+1}\right)+T_{2}^{*}
\end{array}\right),
$$

and

$$
\left(\begin{array}{l}
u_{i+1, j}^{k+1} \\
u_{i, j+1}^{k+1}
\end{array}\right)=\frac{1}{s_{2}}\left(\begin{array}{ll}
s_{1}^{*} & s_{2}^{*} \\
s_{2}^{*} & s_{1}^{*}
\end{array}\right)\left(\begin{array}{c}
\frac{1}{2}\left(r_{2}\right)\left(u_{i, j-1}^{k+1}+u_{i+2, j+1}^{k+1}\right)+\frac{1}{2}\left(r_{1}\right)\left(u_{i+2, j-1}^{k+1}\right)+T_{3}^{*} \\
\frac{1}{2}\left(r_{2}\right)\left(u_{i-1, j}^{k+1}+u_{i+1, j+2}^{k+1}\right)+\frac{1}{2}\left(r_{1}\right)\left(u_{i-1, j+2}^{k+1}\right)+T_{4}^{*}
\end{array}\right),
$$

where,

$$
\begin{aligned}
& S_{1}=\left(1+r_{1}+r_{2}\right)^{2}-1 / 4\left(r_{2}\right)^{2}, \\
& S_{2}=\left(1+r_{1}+r_{2}\right)^{2}-1 / 4\left(r_{1}\right)^{2}, \\
& s_{1}^{*}=s_{1}=1+r_{1}+r_{2}, s_{2}=1 / 2\left(r_{2}\right), s_{2}^{*}=1 / 2\left(r_{1}\right) .
\end{aligned}
$$

The iterative process can be started by either matrix Eqs. (5.2) or (5.3). Once the convergence is attained, the values on the remaining points of the solution domain can be evaluated using FISP formula as described in Section 2.

\section{Numerical experiments and results}

We perform numerical experiments in Mathematica 11 software on a PC of 2GB-RAM to test the capability of the derived schemes FISP, FIRP, FEG, and FEDG in Sections 2, 3, 4, and 5, respectively. The 
various mesh sizes $25,50,75,100$ were assumed in both $x$ and $y$ directions such that $h=\Delta x=\Delta y$ and time step $\tau$ was chosen to be 0.010 throughout the experiment. The relaxation factor is selected as $\omega_{e}=1$ (Gauss-Seidel method), while $l_{\infty}$ norm with tolerance factor $\varepsilon=10^{-5}$ is chosen for convergence criteria. The maximum error of exact $u_{i, j}^{k}$ and numerical $u_{i, j}^{k}$ solution is defined as follows:

$$
E_{\infty}=\max _{0 \leqslant k \leqslant l}\left(\max _{0 \leqslant i, j \leqslant n}\left\{\left|u_{i, j}^{k}-u_{i, j}^{k}\right|\right\}\right),
$$

where, $E_{\infty}$ denotes the maximum error.

Example 6.1. Consider the following time-fractional two dimensional anomalous fractional sub-diffusion equation [13],

$$
\frac{\partial u(x, y, t)}{\partial t}=\frac{\partial^{1-\alpha}}{\partial t^{1-\alpha}}\left[\frac{\partial^{2} u(x, y, t)}{\partial x^{2}}+\frac{\partial^{2} u(x, y, t)}{\partial y^{2}}\right]+f(x, y, t), \quad 0 \leqslant t \leqslant 1
$$

where,

$$
f(x, y, t)=e^{x+y}\left[(1+\alpha) t^{\alpha}-\frac{2 \Gamma(2+\alpha)}{\Gamma(1+2 \alpha)}\right] .
$$

The initial and boundary conditions are given by

$$
\begin{aligned}
& u(x, y, 0)=g(x, y)=0,0<x, y<1, \\
& u(0, y, t)=g_{1}(y, t)=t^{1+\alpha} e^{y}, u(1, y, t)=g_{2}(y, t)=t^{1+\alpha} e^{1+y} \\
& u(x, 0, t)=g_{3}(x, t)=t^{1+\alpha} e^{x}, u(x, 1, t)=g_{4}(x, t)=t^{1+\alpha} e^{1+x}
\end{aligned}
$$

\begin{tabular}{|c|c|c|c|c|c|c|c|c|c|c|c|c|c|}
\hline \multicolumn{2}{|c|}{ Example 1} & \multicolumn{4}{|c|}{$\alpha=0.25$} & \multicolumn{4}{|c|}{$\alpha=0.50$} & \multicolumn{4}{|c|}{$\alpha=0.75$} \\
\hline$h^{-1}$ & Method & Time(s) & Ite. & Ave.Err & Max.Err & Time(s) & Ite. & Ave.Err & Max.Err & Time(s) & Ite. & Ave.Err & Max.Err \\
\hline \multirow{4}{*}{25} & FISP & 119.32 & 69 & $8.8454 \times e^{-2}$ & $9.5767 \times e^{-2}$ & 98.654 & 58 & $6.3380 \times e^{-2}$ & $7.4052 \times e^{-2}$ & 77.934 & 46 & $4.7322 \times e^{-2}$ & $5.2937 \times \mathrm{e}^{-2}$ \\
\hline & FIRP & 77.875 & 42 & $8.4231 \times e^{-2}$ & $9.5667 \times e^{-2}$ & 48.674 & 29 & $6.6743 \times e^{-2}$ & $7.3952 \times e^{-2}$ & 38.874 & 22 & $4.7432 \times e^{-2}$ & $5.2817 \times e^{-2}$ \\
\hline & FEG & 87.534 & 43 & $8.3836 \times e^{-2}$ & $9.5532 \times e^{-2}$ & 56.765 & 30 & $6.8043 \times e^{-2}$ & $7.3872 \times e^{-2}$ & 44.725 & 23 & $4.7432 \times e^{-2}$ & $5.2737 \times e^{-2}$ \\
\hline & FEDG & 44.987 & 23 & $8.3706 \times e^{-2}$ & $9.5405 \times e^{-2}$ & 32.193 & 21 & $6.8373 \times e^{-2}$ & $7.3752 \times e^{-2}$ & 31.953 & 13 & $4.7542 \times e^{-2}$ & $5.2637 \times e^{-2}$ \\
\hline \multirow{4}{*}{50} & FISP & & 111 & $8.7546 \times e^{-2}$ & $9.5375 \times \mathrm{e}^{-2}$ & & 88 & $6.8633 \times e^{-2}$ & $7.3652 \times e^{-2}$ & 133.98 & 76 & $4.7322 \times \mathrm{e}^{-2}$ & $5.2547 \times \mathrm{e}^{-2}$ \\
\hline & FIRP & 84.785 & 67 & $8.7886 \times e^{-2}$ & $9.5255 \times e^{-2}$ & 74.723 & 47 & $6.8443 \times e^{-2}$ & $7.3562 \times e^{-2}$ & 62.730 & 39 & $4.7052 \times e^{-2}$ & $5.2447 \times e^{-2}$ \\
\hline & FEG & 94.098 & 66 & $8.7586 \times e^{-2}$ & $9.5135 \times e^{-2}$ & 84.097 & 47 & $6.8353 \times e^{-2}$ & $7.3412 \times e^{-2}$ & 66.973 & 41 & $4.7432 \times e^{-2}$ & $5.2317 \times e^{-2}$ \\
\hline & FEDG & 55.765 & 31 & $8.7306 \times e^{-2}$ & $9.5055 \times e^{-2}$ & 67.923 & 26 & $6.8345 \times e^{-2}$ & $7.3302 \times e^{-2}$ & 51.849 & 21 & $4.792 \times e^{-2}$ & $5.2257 \times e^{-2}$ \\
\hline \multirow{4}{*}{75} & FISP & & 213 & $7.7056 \times e^{-3}$ & $8.5935 \times e^{-3}$ & & 101 & $5.8433 \times e^{-3}$ & $6.9852 \times e^{-3}$ & & 91 & $3.7742 \times e^{-3}$ & $x e^{-2}$ \\
\hline & FIRP & 133.93 & 97 & $7.7636 \times e^{-3}$ & $8.5855 \times e^{-3}$ & 104.93 & 53 & $5.8933 \times e^{-3}$ & $6.9732 \times e^{-3}$ & 75.492 & 47 & $3.7632 \times e^{-3}$ & $4.3787 \times e^{-3}$ \\
\hline & FEG & 145.77 & 98 & $7.7996 \times e^{-3}$ & $8.5735 \times \mathrm{e}^{-3}$ & 117.67 & 54 & $5.8073 \times e^{-3}$ & $6.9692 \times e^{-3}$ & 79.395 & 48 & $3.7842 \times e^{-3}$ & $4.3647 \times \mathrm{e}^{-3}$ \\
\hline & FEDG & 105.23 & 56 & $7.7426 \times \mathrm{e}^{-3}$ & $8.5685 \times e^{-3}$ & 78.395 & 33 & $5.8713 \times e^{-3}$ & $6.9502 \times e^{-3}$ & 62.874 & 27 & $3.7732 \times \mathrm{e}^{-3}$ & $4.3567 \times e^{-3}$ \\
\hline \multirow{4}{*}{100} & FISP & 398.32 & 342 & $7.7006 \times e^{-2}$ & $8.5585 \times e^{-3}$ & 345.84 & 303 & $5.8883 \times e^{-3}$ & $6.5342 \times e^{-3}$ & 284.942 & 277 & $3.7042 \times e^{-3}$ & $4.3477 \times e^{-3}$ \\
\hline & FIRP & 207.36 & 166 & $7.7846 \times \mathrm{e}^{-3}$ & $8.5475 \times e^{-3}$ & 174.73 & 145 & $5.8633 \times e^{-3}$ & $6.9492 \times e^{-3}$ & 147.986 & 142 & $3.7742 \times \mathrm{e}^{-3}$ & $4.3337 \times e^{-3}$ \\
\hline & FEG & 218.76 & 166 & $7.7256 \times \mathrm{e}^{-3}$ & $8.5375 \times e^{-3}$ & 185.37 & 145 & $5.8673 \times e^{-3}$ & $6.9342 \times e^{-3}$ & 156.307 & 143 & $3.7732 \times e^{-3}$ & $4.3257 \times e^{-3}$ \\
\hline & FEDG & 145.450 & 78 & $7.7546 \times e^{-3}$ & $8.5225 \times e^{-3}$ & 96.521 & 56 & $5.3533 \times e^{-3}$ & $6.9242 \times e^{-3}$ & 126.953 & 64 & $3.7652 \times e^{-3}$ & $4.3177 \times e^{-3}$ \\
\hline
\end{tabular}

The exact analytical solution is $\mathrm{u}(\mathrm{x}, \mathrm{y}, \mathrm{t})=\mathrm{t}^{1+\alpha} \mathrm{e}^{\mathrm{x}+\mathrm{y}}$.

Table 1: Numerical results of FISP, FIRP, FEG, and FEDG methods for different value of $\alpha$ of Example 6.1.

The numerical results are calculated in terms of elapsed time (in second), number of iterations (Ite.), average absolute error (Ave. Error) and maximum absolute error (Max. Error) by FISP, FIRP, FEG and FEDG iterative methods and these are shown in Table 1. In general, results show that the execution timings of FEDG method is only about $(15-24) \%$ of FISP, $(50-75) \%$ of FIRP and $(25-49) \%$ of FEG method. Figure 1 shows the graph of elapsed time of fractional explicit and group iterative methods against the mesh sizes at $\alpha=0.25$, while Figures 2 and 3 illustrate the graph of numerical approximate solution by FEDG method at $\alpha=0.75$. Moreover, since $\tau$ is fixed and as we increases the step size $h$, the maximum error $E_{\infty}$ become significantly small as shown in Table 1. Moreover, $E_{\infty}$ approaches towards zero as $\alpha$ varies from 0 to 1 . 

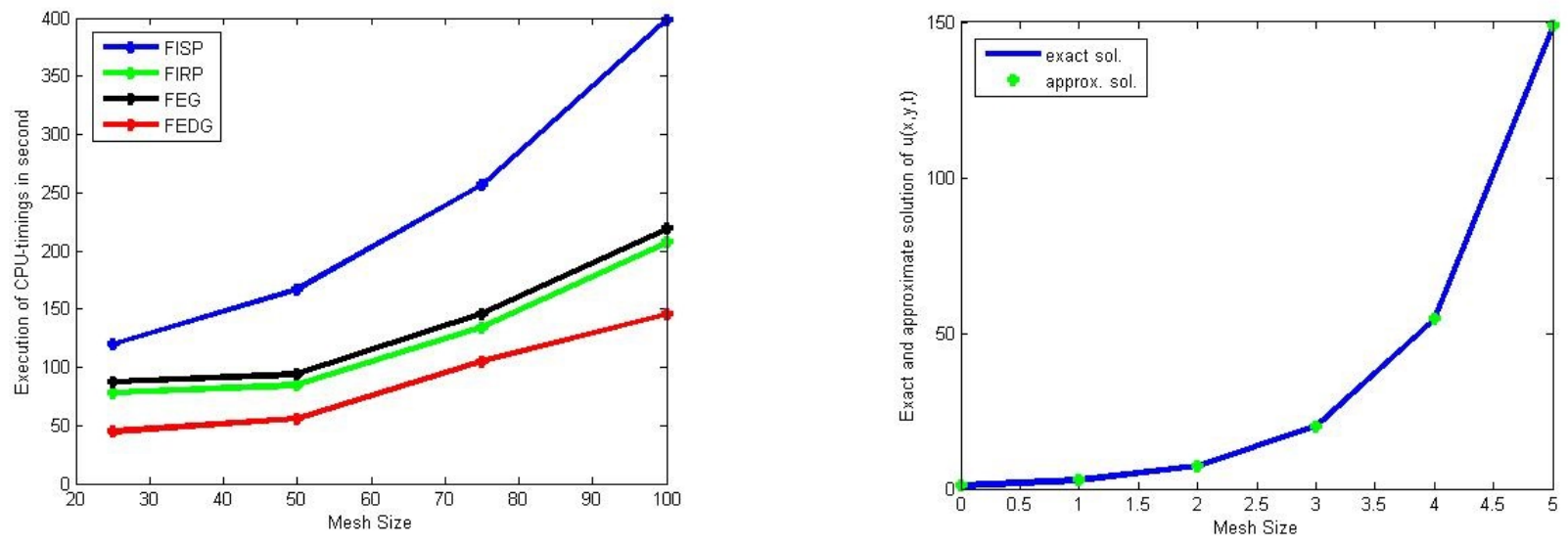

Figure 1: Graph of execution time of fractional explicit point Figure 2: Graph of exact and approximate solution by FEDG and group methods at $\alpha=0.25$. method at $\alpha=0.75$ when $\mathrm{L}=5$.

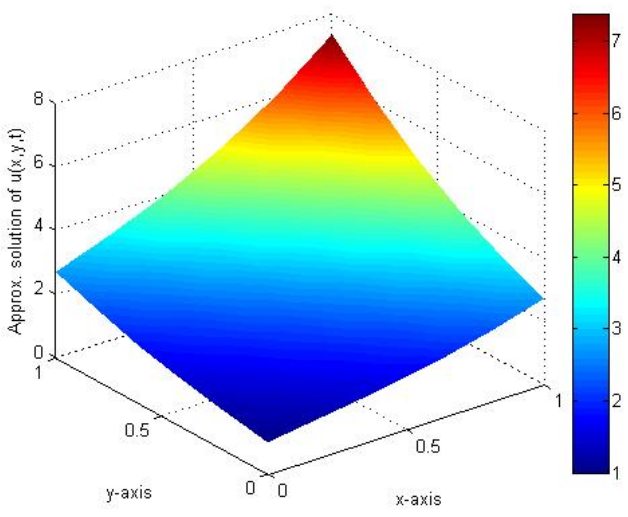

Figure 3: Graph of approximate solution by FEDG method at $\alpha=0.75$.

\section{Conclusion}

In this work, we have successfully derived the point and group iterative schemes in the numerical solution of two-dimensional time-fractional anomalous sub-diffusion equations. The numerical experiment is performed to test the authenticity of the derived iterative schemes. In our findings, we observed that the numerical results obtained from FEDG method are significantly efficient in terms of number of iterations and execution of timings than any other method in this paper. In future, we have several open research problems, specially 2D higher-order compact FDEs and 3D PDEs, on which grouping techniques can be applied.

\section{References}

[1] A. Ali, N. H. M. Ali, On numerical solution of muti-terms fractional differential equations using shifted Chebyshev polynomials, Int. J. Pur. Appl. Math., 120 (2018), 111-125. 1

[2] A. Ali, N. H. M. Ali, On numerical solution of fractional order delay differential equation using Chebyshev collocation method, New Trends in Math. Sci., 6 (2018), 8-17. 1

[3] A. Ali, N. H. M. Ali, Explicit group iterative methods in the solution of two dimensional time fractional diffusion wave equation, Compusoft, 7 (2018), 2931-2938. 1

[4] A. Ali, N. H. M. Ali, New group fractional damped wave iterative solvers using mathematica, International Conference on Mathematical Sciences and Technology (AIP Conference Proceedings, Penang, Malaysia), 2184 (2018), 10 pages. 
[5] A. Ali, N. H. M. Ali, Explicit group iterative methods for the solution of two-dimensional time-fractional telegraph equation, The 4th Innovation and Analytics Conference and Exhibition (AIP Conference Proceedings, Sintok Kedah, Malaysia), 2138 (2019), 6 pages. 1

[6] A. Ali, N. H. M. Ali, On skewed grid point iterative method for solving 2D hyperbolic telegraph fractional differential equation, Adv. Difference Equ., 2019 (2019), 29 pages. 4

[7] M. Amin, M. Abbas, M. K. Iqbal, D. Baleanu, Non-polynomial quintic spline for numerical solution of fourth-order time fractional partial differential equations, Adv. Difference Equ., 2019 (2019), 22 pages. 1

[8] M. Amin, M. Abbas, M. K. Iqbal, A. I. M. Ismial, D. Baleanu, A fourth order non-polynomial quintic spline collocation technique for solving time fractional superdiffusion equations, Adv. Difference Equ., 2019 (2019), 21 pages. 1

[9] N. A. Asif, Z. Hammouch, M. B. Riaz, H. Bulut, Analytical solution of a Maxwell fluid with slip effects in view of the Caputo-Fabrizio derivative, Eur. Phys. J. Plus, 133 (2018), 1-13. 1

[10] A. T. Balasim, N. H. M. Ali, Group iterative methods for the solution of two-dimensional time-fractional diffusion equation, Advances in Industrial and Applied Mathematics (AIP Conference Proceedings, Johor Bahru, Malaysia), 1750 (2016), 7 pages. 1

[11] A. T. Balasim, N. H. M. Ali, The solution of 2-D time-fractional diffusion equation by the fractional modified explicit group iterative method, International Conference on Mathematics, Engineering and Industrial Applications (AIP Conference Proceedings, Songkhla, Thailand), 1775 (2016), 8 pages.

[12] A. T. Balasim, N. H. M. Ali, New group iterative schemes in the numerical solution of the two-dimensional time fractional advection-diffusion equation, Cogent Math., 4 (2017), 19 pages. 1

[13] C.-M. Chen, F. Liu, I. Turner, V. Anh, Numerical schemes and multivariate extrapolation of a two-dimensional anomalous sub-diffusion equation, Numer. Algorithms, 54 (2010), 1-21. 6.1

[14] D. J. Evans, Group explicit iterative methods for solving large linear systems, Int. J. Comput. Math., 17 (1985), 81-108. 1

[15] D. J. Evans, B. Hasan, The numerical solution of the telegraph equation by the alternating group explicit (AGE) method, Int. J. Comput. Math., 80 (2003), 1289-1297. 1

[16] D. J. Evans, M. S. Sahimi, The alternating group explicit iterative method (AGE) to solve the parabolic and hyperbolic partial differential equations, Annual review of numerical fluid mechanics and heat transfer, 2 (1988), 383-389. 1

[17] D. J. Evans, W. S. Yousif, Explicit group iterative methods for solving elliptic partial differential equations in 3-space dimensions, Int. J. Comput. Math., 18 (1986), 323-340. 1

[18] M. M. Ghalib, A. A. Zafar, Z. Hammouch, M. B. Riaz, K. Shabbir, Analytical results on the unsteady rotational flow of fractional-order non-Newtonian fluids with shear stress on the boundary, Discrete Contin. Dyn. Syst., Ser. S, 13 (2020), 683-693. 1

[19] R. Hilfer, Application of fractional Calculus in Physics, World Scientific Publishing Co., River Edge, (2000). 1

[20] N. Khalid, M. Abbas, M. K. Iqbal, Non-polynomial quintic spline for solving fourth-order fractional boundary value problems involving product terms, Appl. Math. Comput., 349 (2019), 393-407. 1

[21] N. Khalid, M. Abbas, M. K. Iqbal, D. Baleanu, A numerical investigation of Caputo time fractional Allen-Cahn equation using redefined cubic B-spline functions, Adv. Difference Equ., 2020 (2020), 22 pages. 1

[22] Y. F. Li, D. L. Wang, Improved efficient difference method for the modified anomalous sub-diffusion equation with a nonlinear source term, Int. J. Comput. Math., 94 (2017), 821-840. 1

[23] K. L. Kew, N. H. M. Ali, New explicit group iterative methods in the solution of three dimensional hyperbolic telegraph equations, J. Comput. Phys., 294 (2015), 382-404. 1

[24] M. B. Riaz, M. A. Imran, K. Shabbir, Analytic solutions of Oldroyd-B fluid with fractional derivatives in a circular duct that applies a constant couple, Alex. Eng. J., 55 (2016), 3267-3275. 1

[25] M. B. Riaz, A. A. Zafar, Exact solutions for the blood flow through a circular tube under the influence of a magnetic field using fractional Caputo-Fabrizio derivatives, Math. Model. Nat. Phenom., 13 (2018), 12 pages. 1

[26] I. M. Sokolov, J. Klafter, From diffusion to anomalous diffusion: acentury after Einsteins Brownian motion, Chaos, 15 (2005), 26-103. 1

[27] P. J. Torvik, R. L. Bagley, On the appearance of the fractional derivative in the behavior of real materials, J. Appl. Mech., 51 (1984), 294-298. 1

[28] P. Zhuang, F. Liu, V. Anh, I. Turne, New solution and analytical techniques of the implicit numerical methods for the anomalous sub-diffusion equation, SIAM J. Numer. Anal., 46 (2008), 1079-1095. 1 